\title{
Ki-67/MIB-1 as a Prognostic Marker in Cervical Cancer - a Systematic Review with Meta-Analysis
}

\author{
Reza Piri ${ }^{1}$, Alireza Ghaffari ${ }^{1 *}$, Nasrin Gholami², Saber Azami-Aghdash ${ }^{3}$, Yasmin \\ PourAli-Akbar $^{4}$, Parviz Saleh ${ }^{5}$, Mohammad Naghavi-Behzad ${ }^{4 *}$
}

\begin{abstract}
Background: In cervical cancer patients it has been reported that there in a significant Ki-67/MIB-1 expression is correlated with survival in cervical cancer patients. However, the prognostic value is still not well understood. Materials and Methods: In the present meta-analysis the prognostic value of Ki-67/MIB-1 with regard to overall survival (OS) and disease-free survival (DFS) in cervical cancer was investigated. The databases of PubMed, ISI Web of Science, Cochrane Central Register of Controlled Trials, EMBASE, Science Direct and Wiley Online Library were used to identify appropriate literature. Results: In order to explore the relationship between Ki-67/ MIB-1 and cervical cancer, we have included 13 studies covering 894 patients in the current meta-analysis. The effect of Ki-67/MIB-1 on OS for pooled random effects HR estimate was 1.63 (95\% confidence interval (CI) 1.09$2.45 ; \mathrm{P}<0.05)$. The pooled HR for DFS was $1.26(95 \% \mathrm{CI} 0.58-2.73 ; \mathrm{P}>0.05)$ and the subgroup analysis indicated Ki-67/MIB1 was associated with DFS (HR=3.67, 95\% CI 2.65-5.09) in Asians. Conclusions: According to this meta-analysis, Ki-67/MIB-1 has prognostic value for OS in patients suffering from cervical cancer. For better evaluation of the prognostic role of Ki-67/MIB-1 on DFS, studies with larger numbers of patients are needed to validate present findings in the future.
\end{abstract}

Keywords: Cervical cancer - Ki-67/MIB-1 - prognosis - meta-analysis

Asian Pac J Cancer Prev, 16 (16), 6997-7002

\section{Introduction}

Cervical cancer is the fourth most common cancer among women. Median age of diagnosis is about 52 years, but has a bimodal distribution with picks at 35 to 39 and 60 to 64 years of age. Annually 500000 death is caused by cervical cancer all around the world. In Europe, a total of 58,373 new cases of cervical cancer and 24,385 deaths were reported in 2012. The overall incidence rate of cervical cancer in Europe is 10.6 per 100,000 (Jemal et al., 2011; Kesic et al., 2012; Ghojazadeh et al., 2013). Surgery, chemotherapy and radiotherapy are the main treatment methods where mostly are used in association with other methods for cervical cancer. However, the long term prognosis of cervical cancer is still poor, with expected 5-year survival rate less than 10\% (Jabbari et al., 2012; Yaghoubi et al., 2012; Amirnia et al., 2014; Eskander and Tewari, 2014).

Cervical cancer is still one of the main problems in women health field worldwide. Even with considering best available therapies, many patients will die due to metastasis or other consequences of cervical cancer (Andrae et al., 2012). Relation between human papilloma virus infection and dysplasia and cervical carcinoma has been known as a scientific fact (Chaturvedi et al., 2011). Human papilloma virus infection in cervical cells' leads to functional changes of host genes and investigating these genes can play an important role in screening and diagnosing cervical cancer (Ghojazadeh et al., 2012a; Ghojazadeh et al., 2012b; Azami-Aghdash et al., 2013; Naghavi-Behzad et al., 2013; Sushma et al., 2014). These functional changes cause changes in cellular cycle which appears through abnormal manifestation of proteins related to this cycle, such as Ki-67 (Ikenberg et al., 2013; Rostamizadeh et al., 2013; Ghojazadeh et al., 2014). Ki-67/MIB-1 is known as predictive factor for tumor development and its expression is correlated with poor prognosis in several types of cancer, such as breast cancer and non-small-cell lung carcinoma (Azambuja et al., 2007; Azami-Aghdash et al., 2013; Rahimi-Ardabili et al., 2012; Fakhrjou et al., 2013; Karami et al., 2013; Mohammadzadeh et al., 2013; Aliasgarzadeh et al., 2014; Shams-Vahdati et al., 2014). Ki-67 status in different tumoral cells and its correlation with prognosis has always been a controversial and significant field of research.

In a study investigating Ki-67 levels and prognosis in

${ }^{1}$ Medical Philosophy and History Research Center, ${ }^{2}$ Hematology and Oncology Research Center, ${ }^{4}$ Students' Research Committee, ${ }^{5}$ Chronic Kidney Diseases Research Center, Tabriz University ofMedical Sciences, Tabriz, ${ }^{3}$ Health Management and Economics Research Center, Iran University of medical sciences, Tehran, Iran*Equal contributors: agh1343@gmail.com *For correspondence: Dr.naghavii@gmail.com 
triple negative breast cancer it had been concluded that, high levels of Ki-67 is associated with more aggressive clinical features, Also it was suggested that Ki-67 can be used as a factor to classify triple negative breast cancer in order to recognize prognostic and response characteristics (Keam et al., 2011). In another study focusing on predicting value of $\mathrm{Ki}-67$ on prognosis in bladder cancer it had been concluded that Ki-67 labeling index may be a precise predictor for poor prognosis in patients with bladder cancer (Sugino et al., 2011). In contrast, according to another study it has been reported that the increased labeling of $\mathrm{Ki}-67$ was not associated with poor outcomes in cervical carcinoma patients of stage I (Van de Putte et al., 2004).

Although lymph node status and pathological features of primary tumor and FIGO stage are thought to be relevant to the prognosis of cervical cancer, considering the fact that the clinical value of $\mathrm{Ki}-67$ for prognostication of cervical cancer is still doubtful, In this meta-analysis it is tried to reveal the impact of $\mathrm{Ki}-67 / \mathrm{MIB}-1$ on overall survival (OS) and/or disease-free survival (DFS) in cervical cancer.

\section{Materials and Methods}

\section{Selecting publications and inclusion criteria}

In this meta-analysis, studies which had investigated the association between Ki-67/MIB-1 and prognosis in cervical cancer, published during 2000-2014. Libraries such as PubMed, ISI Web of Science, EMBASE, Cochrane Central Register of Controlled Trials, ScienceDirect and Wiley Online Library were searched using the following keywords: "cervical cancer", "cervical neoplasm", "prognosis", "Ki-67", "MIB-1", "proliferation", "survival". Inclusion criteria for the studies was composed of: $i$ ) Studies published in English. ii) Studies in which patients with cervical cancer are included. iii) Studies which has investigated correlation between $\mathrm{Ki}-67$ expression and the prognosis in cervical cancer. $i v$ ) Studies with a clear and precise definition of target population (clear inclusion and exclusion criteria). $v$ ) Clearly stated or calculable Hazard ratios (HR) and 95\% CI for OS or DFS. vi) To avoid duplication, If 2 studies had included same samples of patients in several publications, the latest study was chosen.

To extend our search range, the reference list of all identified studies was inspected. All the titles and abstracts of included studies were read by 4 experienced researchers to exclude irrelevant ones. Then, full-texts of the studies were inspected by researchers to see as if the study is included. In case of any disagreements occurred in the suitability of studies, the fifth researcher was included among researchers, then researchers would conduct a discussion until a consensus is finally reached.

\section{Data extraction}

Four authors extracted the required information from publications precisely. The following data were collected from each study: published year, first author's name, number of patients, antibody detecting Ki-67/MIB-1, defined cut-off values, follow-up period, and required data to evaluate the relationship between the expression of Ki-67/MIB-1 and OS and/or DFS. No minimal number of patients was defined to include a study in present metaanalysis, nor a minimal duration of median follow-up. Flow diagram of study is shown in Figure 1. The following exclusion criteria were implemented in all studies: $i$ ) Any research articles but original research articles were excluded. ii) Studies with no control group. iii) Articles with duplicated data. iv) Articles with no usable data.

\section{Statistical methods}

Based on the cut-off points given in the articles by the authors, the expression of Ki-67/MIB-1 was divided into two groups (negative and positive). The expression of Ki-67/MIB-1 on survival was evaluated using HRs to quantitatively aggregate the survival rates. After calculating or extracting HRs and their $95 \%$ confidence intervals (CIs) the relationship of prognosis and $\mathrm{Ki}-67 /$ MIB-1 expression was inspected. The log-rank statistic or its $\mathrm{P}$-value, the HR value estimate and the O-E statistic or its variance was used to recover the estimated HR value and its variance. Based on the outcome provided in the initial publication the HR was evaluated in every study. If any of the mentioned parameters could not be extracted, the case of patients exposed to the risk in each group, the log-rank statistic or the P-value and the whole number of the events were searched to calculate an approximation of the HRs. Engauge Digitizer software was used to extract survival-relative data at stipulated time points from figures. The HRs and its standard error (SE) were reconstructed with filling spreadsheets with HR calculations in order to estimate HRs from published summary statistics (Parmar et al., 1998).

Survival charts were analyzed independently by 4 people to reduce the inaccuracy. If survival was defined for more than two groups (e.g. several cut-off values were used to divide the patients into different groups based on Ki-67/MIB protein expression), we combined the data of some groups in order that we can make a comparison between two groups. For each study, the OS and/or DFS were analyzed. Considering subgroups such as ethnicity (Africans, Europeans or Asians) and various treatments (surgery and RT) HR was calcualted.

All the data analyses were performed with the software of STATA 8.2 (StataCorp, College Station, Tex). Using the method reported by Yusuf S et al, the individual HRs were combined into an overall HR (Yusuf et al., 1985). Statistical heterogeneity was measured using the Q statistic ( $p<0.10$ was set as representative of significant statistical heterogeneity). A random-effects model was used if the Q-tests implied that there existed heterogeneity between the studies, Also subgroup analysis was used to investigate any source of heterogeneity. The publication biases was estimated using Begg's funnel plot. If $P$ value was $>0.05$, it was confirmed that no publication biases was found.

\section{Results}

Literature search and characteristics of the included studies 
Ki-67/MIB-1 as a Prognostic Marker in Cervical Cancer-a Systematic Review with Meta-Analysis

Table 1. Main Characteristics of All Studies Included in the Meta-Analysis (Overall Survival)

\begin{tabular}{|c|c|c|c|c|c|}
\hline First Author(publication year) & $\begin{array}{c}\text { Total } \\
\text { patients }\end{array}$ & $\begin{array}{l}\text { Median follow-up } \\
\text { (months) }\end{array}$ & Antibody & Threshold & $\begin{array}{l}\text { Hazard ratio } \\
(95 \% \mathrm{CI})\end{array}$ \\
\hline Bahnassy AA (2007) & 43 & NR & MIB-1 & $1 \%(\mathrm{PI})$ & $1.53(1.03-20.7)$ \\
\hline Ho DM (2000) & 97 & 83.4 (mean) & MIB-1 & $55 \%(\mathrm{LI})$ & $3.62(1.3-10.09)$ \\
\hline Klimek M (2011) & 122 & $5 y$ & Anti-Ki-67 & $52 \%(\mathrm{PI})$ & $2.28 \quad(1.27-4.11)$ \\
\hline Oka K (2000) & 75 & NR & Anti-MIB-1 & $40 \%(\mathrm{PI})$ & $3.4 \quad(0.53-22.15)$ \\
\hline Shiohara SI (2005) & 103 & 65.1 (mean) & Anti-Ki-67 & $50 \%(\mathrm{PI})$ & $1.57 \quad(0.53-4.66)$ \\
\hline Shiohara SII (2005) & 103 & 65.1 (mean) & Anti-Ki-67 & $50 \%(\mathrm{PI})$ & $1.44 \quad(0.32-6.39)$ \\
\hline Sultana H (2003) & 30 & $5 y$ & Anti-Ki-67 & $33 \%(\mathrm{LI})$ & $0.46 \quad(0.04-5.99)$ \\
\hline Suzuki M (2000) & 67 & 78 & Anti-MIB-1 & $26.4 \%$ (PI) & $0.49 \quad(0.15-2.53)$ \\
\hline Yang M (2014) & 180 & 64 (mean) & Anti-Ki-67 & $10 \%(\mathrm{PI})$ & $3.80 \quad(1.80-4.70)$ \\
\hline Zhang T (2012) & 40 & NR & Anti-Ki-67 & $34.62 \%(\mathrm{PI})$ & $3.44 \quad(0.7-18.34)$ \\
\hline Zhang T (2012) & 48 & NR & Anti-Ki-67 & $32.74 \%(\mathrm{PI})$ & $3.04(0.73-13.26)$ \\
\hline
\end{tabular}

CI: confidence interval; NR: not reported; y: years. PI: proliferation index; LI: labeling index

Table 2. Main Features of All Studies Included in the Meta-Analysis (Disease-Free Survival)

\begin{tabular}{|c|c|c|c|c|c|}
\hline $\begin{array}{l}\text { First Author } \\
\text { (publication year) }\end{array}$ & $\begin{array}{c}\text { Total } \\
\text { patients }\end{array}$ & $\begin{array}{l}\text { Median follow-up } \\
\text { (months) }\end{array}$ & Antibody & Threshold & $\begin{array}{l}\text { Hazard ratio } \\
(95 \% \mathrm{CI})\end{array}$ \\
\hline Bahnassy AA(2006) & 38 & 13(mean) & NR & NR & $1.38(0.66-2.87)$ \\
\hline Graflund MIN+(2002) & 37 & 222(mean) & MIB-1 & $1 \%(\mathrm{PI})$ & $0.30(0.10-0.91)$ \\
\hline Graflund MIIN+(2002) & 37 & 222(mean) & MIB-1 & $1 \%(\mathrm{PI})$ & $0.28(0.01-0.85)$ \\
\hline Klimek M(2011) & 122 & $5 y$ & Anti-Ki-67 & $52 \%(\mathrm{PI})$ & $1.19(1.05-3.50)$ \\
\hline Oka K(2000) & 75 & NR & Anti-MIB-1 & $40 \%(\mathrm{PI})$ & $4.88(0.42-55.98)$ \\
\hline Yang M(2014) & 180 & 64(mean) & Anti-Ki-67 & $10 \%(\mathrm{PI})$ & $3.80(2.50-4.90)$ \\
\hline
\end{tabular}

CI: confidence interval; NR: not reported; y: years; PI: proliferation index

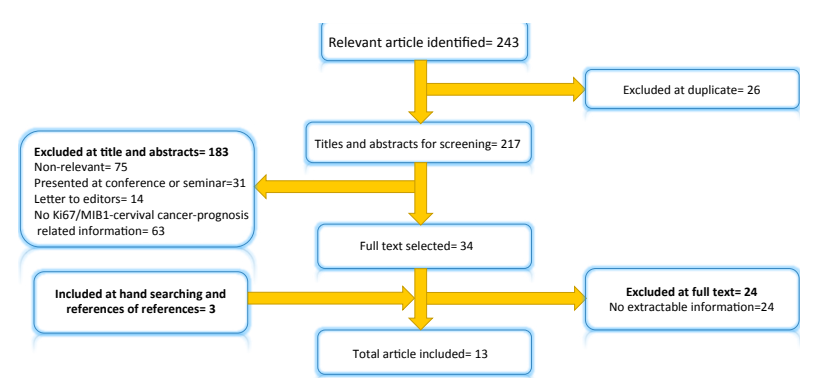

Figure 1. Flow Diagram of Studies Included in the Meta-Analysis

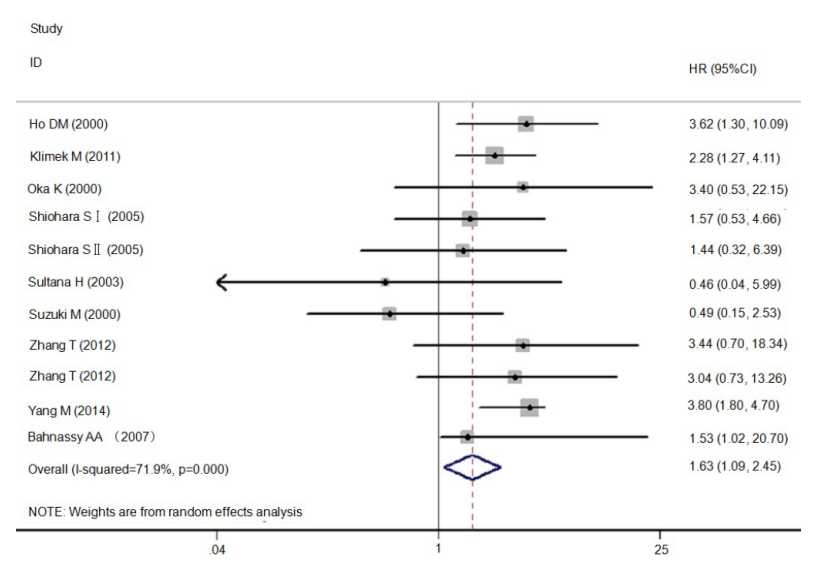

Figure 2. Results of the Meta-Analysis with All Evaluable Studies for Overall Survival

In present study 13 studies including 894 patients were identified, which had been published between 2000 and 2014 (Ho et al., 2000; Oka et al., 2000; Suzuki et al., 2000; Graflund et al., 2002; Sultana et al., 2003; Shiohara

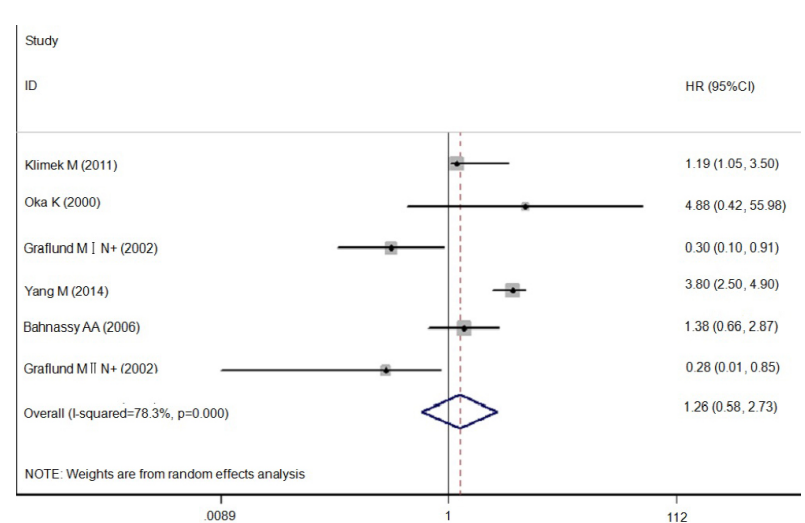

Figure 3. Results of the Meta-Analysis With All Evaluable Studies For Disease Free Survival

et al., 2005; Bahnassy et al., 2006; Bahnassy et al., 2007; Klimek et al., 2011; Zhang et al., 2012a; Zhang et al., 2012b; Yang et al., 2014). All studies had used indirect hemaggultination reaction to detect antibodies. Anti-Ki67 and MIB-1 were applied to detect the Ki-67 expression. Main characteristics of included studies for met-analysis of OS and DFS are shown in Table 1 and 2, respectively. Subgroup analyses were performed in all 8 studies with ethnicity and treatment.

\section{Meta-analysis}

The meta-analysis results of OS is shown in Figure 2. Eleven studies used immunohistochemistry (IHC) for OS, the pooled random HR was $1.63(95 \%$ CI 1.09$2.45 ; \mathrm{P}<0.05)$, with significant evidence of heterogeneity between studies $\left(\mathrm{I}^{2}=71.9 \%, \mathrm{Q}=2.36, \mathrm{P}<0.05\right)$. Restricting analysis to ethnicity, data failed to show a correlation in 


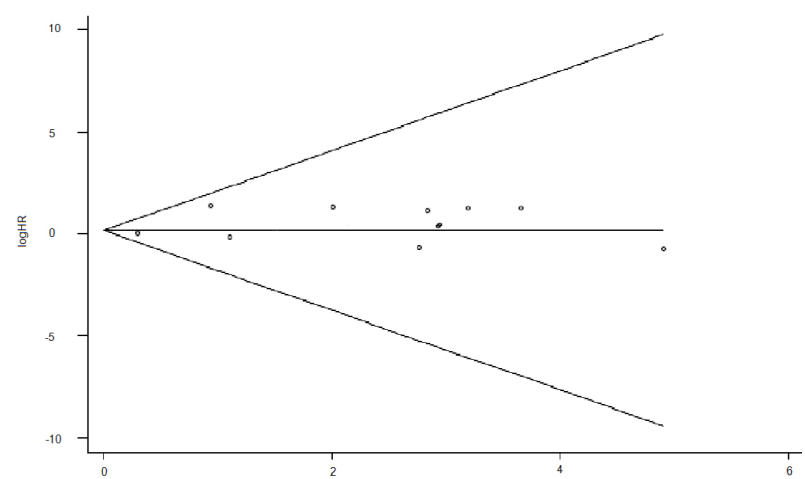

Figure 4. Begg Test was Performed with Pseudo $95 \%$ Confidence Limits to Detect the Publication Bias Risk of Overall Survival $(P=0.907)$

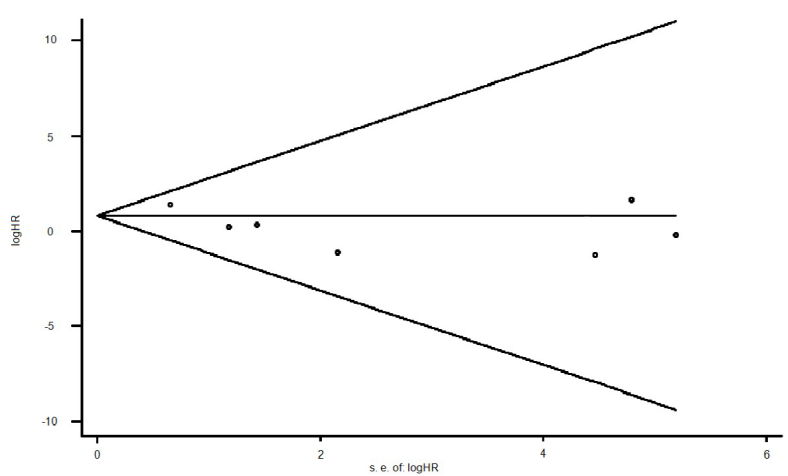

Figure 5. Begg Test was Performed with Pseudo 95\% Confidence Limits to Detect the Publication Bias Risk of DFS ( $P=0.014)$

Africans $(\mathrm{HR}=1.53,95 \% \mathrm{CI}$ 0.34-6.86) and Europeans ( $\mathrm{HR}=1.29,95 \% \mathrm{CI}$ 0.74-2.23). But statistically difference was observed in Asians ( $\mathrm{HR}=1.53$, 95\%CI 1.04-3.23). By treatment subgroup analysis, no dramatic differences were discovered in surgery $(\mathrm{HR}=1.97,95 \% \mathrm{CI} 0.78$ $4.99)$ or RT treatment (HR=1.56, 95\%CI 0.93-2.63). The meta-analysis results of DFS is shown in Figure 3. Seven studies assessed DFS with the pooled random HR of 1.26 (95\%CI 0.58-2.73; P>0.05) and there was evidence for heterogeneity $\left(\mathrm{I}^{2}=78.3 \%, \mathrm{Q}=0.59, \mathrm{P}>0.05\right)$. Altogether, six studies were assessed ethnicity. The subgroup analysis revealed that Asians was associated with DFS (HR=3.67, 95\% CI 2.65-5.09). Whereas, no association was found in Europeans (HR=0.81, 95\%CI 0.39-1.67). Of 5 studies eligible for treatment subgroup, analysis of these data showed that DFS was not positively associated with surgery $(\mathrm{HR}=1.13,95 \% \mathrm{CI} 0.71-2.24)$ and $\mathrm{RT}(\mathrm{HR}=1.26$, 95\% CI 0.71-2.24).

To evaluate the meta-results stability sensitivity analyses were performed. For OS and DFS, the results indicated that random effects estimate before and after the deletion studies were similar. Also publication bias statistics were determined by using the method of Begg's test. No publication biases were found in $11 \mathrm{OS}$ on studies $(\mathrm{P}=0.907)$ (Figure 4); But significant difference in 6 studies on DFS ( $\mathrm{P}=0.014)$ (Figure 5).

\section{Discussion}

As far as Ki-67/MIB-1 is closely integrated with cell proliferation, it has been known as a tumor marker in cancers due to its close correlation with cell proliferation. It has been concluded that $\mathrm{Ki}-67 / \mathrm{MIB}-1$ expression is associated with poor prognosis (Ho et al., 2000; Oka et al., 2000; Suzuki et al., 2000; Costa et al., 2001; Sultana et al., 2003; Bahnassy et al., 2007; Klimek et al., 2011; Zhang et al., 2012a; Zhang et al., 2012b; Yang et al., 2014). Based on results of present study Ki-67/MIB-1 can be used as a predictor of prognosis because of its profound correlation with prognosis.

Detailed cell cycle analysis has revealed that $\mathrm{Ki}-67 /$ MIB-1 is present in the nuclei of cells in the G1, S, G2 and mitosis phases. However, the mechanism for the Ki-67 gene expression is still unknown. Although some studies has revealed possible or partial mechanisms responsible for Ki-67/MIB-1 expression; for example in a study it was proven that $\mathrm{p} 53$-binding motifs intervened part of the transcriptional suppression of $\mathrm{Ki}-67$ promoter through an interaction with p53, also p53 was able to suppress the Sp1-stimulated Ki-67 promoter activity (Wang et al., 2011).

There has been many studies investigating the relationship between Ki67/MIB-1 expression and prognosis in different types of cancers; such as ovarian cancer (Battista et al., 2014), breast cancer (Goldhirsch et al., 2013), esophageal squamous cell carcinoma (Hu et al., 2011), lung cancer (Warth et al., 2014), colorectal cancer (Peng et al., 2013), glioblastoma (Smith et al., 2012) and prostate cancer (Tollefson et al., 2014). All mentioned studies indicated that which indicated that Ki-67/MIB-1 expression was associated with poor prognosis so it can be used as a prognostic predictor might be used as a potential predictor for the survival of patients with some cancers.

No biases were found in OS studies, while it was shown that there were biases for DFS (Figure 4 and 5). The main point which might have affected present metaanalysis is strict exclusion criteria for this study, for example by excluding non-English studies many studies bearing prominent results were excluded.

The techniques used to detect the expression of Ki-67 /MIB-1 might have been another source of bias. In present meta-analysis, not always the antibody used to stain $\mathrm{Ki}$ 67 were the same (Table 1 and 2). It has been shown that using equivalent antibodies appeared to make differences in variable staining features (Lindboe and Torp, 2002; Lindboe et al., 2003). It has been proven that MIB-1 antibody is the most suitable antibody to detect Ki-67, because it has the highest sensitivity and give the clearest staining when compared to other available antibodies (Fasanella et al., 2011; Lindboe et al., 2003). Also a positive ki67 staining of the tumor cells was confirmed when it was compare with positive nuclear staining for a standard sample. So using different antibodies and a protocol to count the stained cells without a confirmed standard may lead to a variance in results.

Another potential source of biases, might be due to HR variations resulted from extrapolation of survival-relative data when no HR existed, using Kaplan-Meier model. Although for certain articles HR was directly extracted using the original data provided in study. Considering this 
as a potential source of bias, four independent persons analyzed the data extracted from Kaplan-Meier model, then extrapolated the HR to reduce variability. Then estimated HR were compared with the original results, but no variances were found.

In present meta-analysis, different cut-off points were used to apply it in definition of cervical cancer with a positive Ki-67/MIB-1. It is difficult to determine a standard critical cut-off values in clinical practice, because sometimes median or arbitrary cut-off value are selected. It has been already confirmed that using minimum P-value approach to select cut-off value may result in significant biases in conclusions (Altman et al., 1994). Ki67 labelling indices tend to cluster around values ending with 5 or 0 both in cases where the values were acquired by counting the fraction of stained tumor cell nuclei by estimation (Cserni et al., 2014). Although it seems more standard for analysis of prognostic markers to define the expression levels based on median, but it might lead to loss of information (Altman et al., 1994). In a study investigating role of $\mathrm{Ki}-67$ proliferative index in predicting overall short-term survival in patients with typical and atypical pulmonary carcinoid tumors it was concluded that Ki67 index cut-off value of 5\% can perform best for predicting overall survival while satisfying both specificity and sensitivity (Walts et al., 2012). In another study about breast cancer (Spyratos et al., 2002) using different Ki-67/MIB-1 cut-offs, it was concluded that a Ki-67/MIB-1 cut-off of $10 \%$ was optimal to classify tumor (high and low proliferation indexes) in therapeutic trials. Also a Ki-67/MIB-1 cut-off of 25\% was optimal to detect highly proliferative tumors (Spyratos et al., 2002). In summary, a suitable threshold is still needed for Ki-67/ MIB-1 to certify for cervical cancer.

Except Ki-67/MIB-1 positivity status, other features of disease may influence the survival; such as tumoral cell grade, metastasis, lymph node involvement. Unfortunately in present study just the univariate prognostic value of Ki-67/MIB-1 was investigated. So it is really difficult to jump to conclusions before considering other factors. In order to overcome this limitation prospective studies with larger population are needed.

In conclusion, $\mathrm{Ki}-67 / \mathrm{MIB} 1$ is considered as a prognostic marker in cervical cancer. According to the results that patients with high Ki-67/MIB1 expression had significantly less OS than patients with low expression of $\mathrm{Ki}-67 / \mathrm{MIB} 1 \quad(\mathrm{P}<0.001)$. Although considering possible sources of biases it is necessary that studies with programmed design to overcome biases needs to be conducted, to offer a better conclusion about the relationship between $\mathrm{Ki}-67 / \mathrm{MIB} 1$ and prognosis of patients with cervical cancer.

\section{Acknowledgements}

This study was supported by Tabriz University of Medical Sciences.

\section{References}

Aliasgarzadeh A, Ghojazadeh M, Haji-Hoseini R, et al (2014).
Age related secretary pattern of growth hormone, insulinlike growth factor-I \& insulin-like growth factor binding protein-3 in postmenopausal women. Indian J Med Res, 139, 598-602.

Altman DG, Lausen B, Sauerbrei W, et al (1994). Dangers of using "optimal" cutpoints in the evaluation of prognostic factors. J Natl Cancer Inst, 86, 829-35.

Amirnia M, Babaie-Ghazani A, Fakhrjou A, et al (2014). Immunohistochemical study of cyclooxygenase-2 in skin tumors. J Dermatolog Treat, 25, 380-7.

Andrae B, Andersson TM, Lambert PC, et al (2012). Screening and cervical cancer cure: population based cohort study. $B m j, 344$.

Azami-Aghdash S, Ghaffari S, Sadeghi-Bazargani H, et al (2013). Developing Indicators of Service Quality Provided for CardiovascularPatients Hospitalized in Cardiac Care Unit. J Cardiovascular Thoracic Res, 5, 23.

Azami-Aghdash S, Ghojazadeh M, Pournaghi Azar F, et al (2013). Fluoride concentration of drinking waters and prevalence of fluorosis in iran: a systematic review. $J$ Dent Res Dent Clin Dent Prospects, 7, 1-7.

Bahnassy AA, Zekri AR, Alam El-Din HM, et al (2006). The role of cyclins and cyclins inhibitors in the multistep process of HPV-associated cervical carcinoma. J Egypt Natl Canc Inst, 18, 292-302.

Bahnassy AA, Zekri AR, Saleh M, et al (2007). The possible role of cell cycle regulators in multistep process of HPVassociated cervical carcinoma. BMC Clin Pathol, 7, 4.

Battista MJ, Mantai N, Sicking I, et al (2014). Ki-67 as an independent prognostic factor in an unselected cohort of patients with ovarian cancer: Results of an explorative, retrospective study. Oncol Reports, 31, 2213-9.

Chaturvedi AK, Engels EA, Pfeiffer RM, et al (2011). Human papillomavirus and rising oropharyngeal cancer incidence in the United States. J Clin Oncol, 29, 4294-301.

Costa S, Terzano P, Santini D, et al (2001). Neoadjuvant chemotherapy in cervical carcinoma: regulators of cell cycle, apoptosis, and proliferation as determinants of response to therapy and disease outcome. Am J Clin Pathol, 116, 729-37.

Cserni G, Vörös A, Liepniece-Karele I, et al (2014). Distribution pattern of the Ki67 labelling index in breast cancer and its implications for choosing cut-off values. Breast, 23, 259-63.

de Azambuja E, Cardoso F, de Castro G, Jr, et al (2007). Ki-67 as prognostic marker in early breast cancer: a meta-analysis of published studies involving 12,155 patients. Br J Cancer, 96, 1504-13.

Eskander RN, Tewari KS (2014). Beyond angiogenesis blockade: targeted therapy for advanced cervical cancer. J Gynecol Oncol, 25, 249-59.

Fakhrjou A, Dastranj-Tabrizi A, Ghojazadeh M, et al (2013). Diagnostic value of protein Ki67 (MIB-1) in atypical pap smears of postmenopausal women. Asian Pac J Cancer Prev, 14, 4815-8.

Fasanella S, Leonardi E, Cantaloni C, et al (2011). Proliferative activity in human breast cancer: Ki-67 automated evaluation and the influence of different Ki-67 equivalent antibodies. Diagn Pathol, 6, 7.

Ghojazadeh M, Azar ZF, Saleh P, et al (2012a). Knowledge and attitude of Iranian university students toward human papilloma virus. Asian Pac J Cancer Prev, 13, 6115-9.

Ghojazadeh M, Mohammadi M, Azami-Aghdash S, et al (2013). Estimation of cancer cases using capture-recapture method in Northwest Iran. Asian Pac J Cancer Prev, 14, 3237-41.

Ghojazadeh M, Naghavi-Behzad M, Azar ZF, et al (2012b). Parental knowledge and attitudes about human papilloma virus in Iran. Asian Pac J Cancer Prev, 13, 6169-73.

Ghojazadeh M, Naghavi-Behzad M, Nasrolah-Zadeh R, et al 
(2014). Knowledge production status of Iranian researchers in the gastric cancer area: based on the medline database. Asian Pac J Cancer Prev, 15, 5083-8.

Goldhirsch A, Winer E, Coates A, et al (2013). Personalizing the treatment of women with early breast cancer: highlights of the St Gallen International Expert Consensus on the Primary Therapy of Early Breast Cancer 2013. Ann Oncol, 24, 2206-23.

Graflund M, Sorbe B, Karlsson M (2002). MIB-1, p53, bcl-2, and WAF-1 expression in pelvic lymph nodes and primary tumors in early stage cervical carcinomas: correlation with clinical outcome. Int J Oncol, 20, 1041-7.

Ho DM, Hsu CY, Chiang H (2000). MIB-1 labeling index as a prognostic indicator for survival in patients with FIGO stage IB squamous cell carcinoma of the cervix. Gynecol Oncol, 76, 97-102.

$\mathrm{Hu}$ Y, Correa AM, Hoque A, et al (2011). Prognostic significance of differentially expressed miRNAs in esophageal cancer. Int J Cancer, 128, 132-43.

Ikenberg H, Bergeron C, Schmidt D, et al (2013). Screening for cervical cancer precursors with p16/Ki-67 dual-stained cytology: results of the PALMS study. J Natl Cancer Inst, 105, 1550-7.

Jabbari H, Alikhah H, Alamdari NS, et al (2012). Developing the use of quality indicators in sterilization practices. Iranian $J$ Health, 41, 64.

Jemal A, Bray F, Center MM, et al (2011). Global cancer statistics. Ca Cancer J Clin, 61, 69-90.

Karami H, Baradaran B, Esfahani A, et al (2013). siRNAmediated silencing of survivin inhibits proliferation and enhances etoposide chemosensitivity in acute myeloid leukemia cells. Asian Pac J Cancer Prev, 14, 7719-24.

Keam B, Im S-A, Lee K-H, et al (2011). Ki-67 can be used for further classification of triple negative breast cancer into two subtypes with different response and prognosis. Breast Cancer Res, 13, 22.

Kesic V, Poljak M, Rogovskaya S (2012). Cervical cancer burden and prevention activities in Europe. Cancer Epidemiol Biomarkers Prev, 21, 1423-33.

Klimek M, Kruczak A, Rys J, et al (2011). Clinico-morphological parameters affecting survival of patients with advanced cervical cancer. Pol J Pathol, 62, 250-6.

Lindboe CF, Torp SH (2002). Comparison of Ki-67 equivalent antibodies. J Clin Pathol, 55, 467-71.

Lindboe CF, von der Ohe G, Torp SH (2003). Determination of proliferation index in neoplasms using different Ki-67 equivalent antibodies. APMIS, 111, 567-70.

Mohammadzadeh M, Pourzand A, Eftekhar-Sadat AT, et al (2013). A case of concurrent several forms of thyroid cancer. Niger Med J, 54, 351-3.

Naghavi-Behzad M, Alizadeh M, Azami S, et al (2013). Risk Factors of Congenital Heart Diseases: Case-Control Study in Northwest Iran. J Cardiovascular Thoracic Rese, 5, 5.

Oka K, Suzuki Y, Nakano T (2000). High growth fraction at 9 grays of radiotherapy is associated with a good prognosis for patients with cervical squamous cell carcinoma. Cancer, 89, 1526-31.

Parmar MK, Torri V, Stewart L (1998). Extracting summary statistics to perform meta-analyses of the published literature for survival endpoints. Stat Med, 17, 2815-34.

Peng Y, Wang L, Gu J (2013). Elevated preoperative carcinoembryonic antigen (CEA) and Ki67 is predictor of decreased survival in IIA stage colon cancer. World J Surg, 37, 208-13.

Rahimi-Ardabili B, Argani H, Ghorbanihaghjo A, et al (2012). Paraoxonase enzyme activity is enhanced by zinc supplementation in hemodialysis patients. Renal Failure,

\section{4, 1123-8.}

Rostamizadeh L, Fakhrjou A, Montazeri V, et al (2013). Bcl-2 gene expression in human breast cancers in iran. Asian Pac J Cancer Prev, 14, 4209-14.

Shams-Vahdati S, Vand-Rajavpour Z, Paknezhad S-P, et al (2014). Cost-effectiveness of cardiac biomarkers as screening test in acute chest pain. J Cardiovasc Thoracic Res, 6, 29.

Shiohara S, Shiozawa T, Miyamoto T, et al (2005). Expression of cyclins, p53, and Ki-67 in cervical squamous cell carcinomas: overexpression of cyclin $\mathrm{A}$ is a poor prognostic factor in stage Ib and II disease. Virchows Arch, 446, 626-33.

Smith SJ, Tilly H, Ward JH, et al (2012). CD105 (Endoglin) exerts prognostic effects via its role in the microvascular niche of paediatric high grade glioma. Acta Neuropathol, 124, 99-110.

Spyratos F, Ferrero-Pous M, Trassard M, et al (2002). Correlation between MIB-1 and other proliferation markers: clinical implications of the MIB-1 cutoff value. Cancer, 94, 2151-9.

Sugino T, Baba K, Hoshi N, et al (2011). Overexpression of fatty acid synthase in human urinary bladder cancer and combined expression of the synthase and $\mathrm{Ki}-67$ as a predictor of prognosis of cancer patients. Med Mol Morphol, 44, 146-50.

Sultana H, Kigawa J, Kanamori Y, et al (2003). Chemosensitivity and p53-Bax pathway-mediated apoptosis in patients with uterine cervical cancer. Ann Oncol, 14, 214-9.

Sushma M, Vamsikrishna B, Babu M, et al (2014). A review on role of human papilomma virus (HPV) in cervical cancer. PharmaTutor, 2, 21-30.

Suzuki M, Tsukagoshi S, Saga Y, et al (2000). Assessment of proliferation index with MIB-1 as a prognostic factor in radiation therapy for cervical cancer. Gynecol Oncol, 79, 300-4.

Tollefson MK, Karnes RJ, Kwon ED, et al (2014). Prostate cancer Ki-67 (MIB-1) expression, perineural invasion, and gleason score as biopsy-based predictors of prostate cancer mortality: the mayo model. Mayo Clinic Proc, 89, 308-18.

Van de Putte G, Kristensen GB, Lie AK, et al (2004). Cyclins and proliferation markers in early squamous cervical carcinoma. Gynecol Oncol, 92, 40-6.

Walts AE, Ines D, Marchevsky AM (2012). Limited role of Ki-67 proliferative index in predicting overall short-term survival in patients with typical and atypical pulmonary carcinoid tumors. Mod Pathol, 25, 1258-64.

Wang M-J, Pei D-S, Qian G-W, et al (2011). p53 regulates Ki-67 promoter activity through $\mathrm{p} 53$-and $\mathrm{Sp} 1$-dependent manner in HeLa cells. Tumor Biol, 32, 905-12.

Warth A, Cortis J, Soltermann A, et al (2014). Tumour cell proliferation (Ki-67) in non-small cell lung cancer: a critical reappraisal of its prognostic role. Br J Cancer, 111, 1222-9.

Yaghoubi A, Tabrizi JS, Mirinazhad MM, et al (2012). Quality of life in cardiovascular patients in Iran and factors affecting it: A systematic review. J Cardiovascular Thoracic Res, 4, 95.

Yang M, Liu YD, Wang YY, et al (2014). Ubiquitin-specific protease 22: a novel molecular biomarker in cervical cancer prognosis and therapeutics. Tumour Biol, 35, 929-34.

Yusuf S, Peto R, Lewis J, et al (1985). Beta blockade during and after myocardial infarction: an overview of the randomized trials. Prog Cardiovasc Dis, 27, 335-71.

Zhang T, Zhao C, Chen D, et al (2012a). Overexpression of AQP5 in cervical cancer: correlation with clinicopathological features and prognosis. Med Oncol, 29, 1998-2004.

Zhang T, Zhao C, Luo L, et al (2012b). The expression of Mcl-1 in human cervical cancer and its clinical significance. Med Oncol, 29, 1985-91. 